\title{
Charm baryon production and fragmentation fractions in pp collisions with ALICE
}

\section{Jinjoo Seo* on behalf of the ALICE collaboration}

Inha University, 22212, Incheon, Republic of Korea

E-mail: jin.joo.seo@cern.ch

In this contribution, the latest measurements of $\Lambda_{\mathrm{c}}^{+}, \Xi_{\mathrm{c}}^{0,+}, \Sigma_{\mathrm{c}}^{0,++}$, and the first measurement of $\Omega_{\mathrm{c}}^{0}$ baryons performed with the ALICE detector at midrapidity in pp collisions at $\sqrt{s}=5.02$ and $13 \mathrm{TeV}$ are presented. In addition, the $\Lambda_{\mathrm{c}}^{+}$measurement down to $p_{\mathrm{T}}=0$ in $\mathrm{p}-\mathrm{Pb}$ collisions will be discussed. Finally, the first measurements of the total charm cross section per unit of rapidity and the fragmentation fractions at midrapidity in pp collisions at the LHC including the charm baryons are discussed.

*** 10th International Workshop on Charm Physics (CHARM2020) ***

*** 31 May - 4 June, 2021 ***

*** Mexico City, Mexico - Online ***

${ }^{*}$ Speaker 


\section{Introduction}

Recent measurements of charm-baryon production at midrapidity performed by ALICE in pp and $\mathrm{p}-\mathrm{Pb}$ collisions $[1,2]$ showed a baryon-to-meson ratio significantly higher than that measured in $\mathrm{e}^{+} \mathrm{e}^{-}$collisions [3,4]. This enhancement cannot be explained by model predictions considering only charm fragmentation fractions obtained in $\mathrm{e}^{+} \mathrm{e}^{-}$collisions and suggests that the parton-tohadron fragmentation of charm quark is not universal across different collision systems. Therefore, measurements of charm-baryon production are crucial to investigate the hadronisation mechanism of charm quarks in pp collisions.

The studies of charm hadrons in small systems can be used as a baseline to understand the properties of the quark-gluon plasma (QGP). The measurements of charm-baryons in pp collisions provide the necessary reference for measurements in $\mathrm{Pb}-\mathrm{Pb}$ collisions and allow us to test $\mathrm{pQCD}$ predictions. The measurements of charm-baryon in $\mathrm{p}-\mathrm{Pb}$ collisions provide essential information about Cold Nuclear Matter (CNM) effects to understand the possible presence of collective effects or modification of hadronisation mechanisms [5].

\section{Results}

\subsection{Charm baryon production}

ALICE has measured all single-charm hadron ground states in pp collisions, including charm mesons $\left(\mathrm{D}^{0}, \mathrm{D}^{+}, \mathrm{D}_{\mathrm{s}}^{+}\right)$at $\sqrt{s}=5.02 \mathrm{TeV}[6]$ and charm baryons $\left(\Lambda_{\mathrm{c}}^{+}, \Xi_{\mathrm{c}}^{0,+}\right.$ and $\left.\Omega_{\mathrm{c}}^{0}\right)$ at $\sqrt{s}=5.02 \mathrm{TeV}$ and $\sqrt{s}=13 \mathrm{TeV}[2,7]$. In addition, ALICE measured $\Lambda_{\mathrm{c}}^{+}$in $\mathrm{p}-\mathrm{Pb}$ collisions at $\sqrt{s_{\mathrm{NN}}}=5.02 \mathrm{TeV}$ down to $p_{\mathrm{T}}=0$.

The $\Lambda_{\mathrm{c}}^{+} / \mathrm{D}^{0}$ ratios in pp and $\mathrm{p}-\mathrm{Pb}$ collisions at $\sqrt{s_{\mathrm{NN}}}=5.02 \mathrm{TeV}$ are shown in the left panel of Fig.1. At low $p_{\mathrm{T}}$, the $\Lambda_{\mathrm{c}}^{+} / \mathrm{D}^{0}$ ratio measured in $\mathrm{p}-\mathrm{Pb}$ collisions is significantly lower than in pp collisions, and at intermediate $p_{\mathrm{T}}$, the $\Lambda_{\mathrm{c}}^{+} / \mathrm{D}^{0}$ ratio measured in $\mathrm{p}-\mathrm{Pb}$ collisions is higher with respect to the one measured in pp collisions. This modification can be interpreted as due to the radial flow or additional hadronisation process in $\mathrm{p}-\mathrm{Pb}$ collisions. The $\Sigma_{\mathrm{c}}^{0,++} / \mathrm{D}^{0}$ ratio measured in pp collisions at $\sqrt{s}=13 \mathrm{TeV}$ is shown in the right panel of Fig.1. The model calculation from PYTHIA8 Monash [8] largely underestimates the measurement, the statistical hadronisation model (SHM) with excited charm baryon states predicted by the relativistic quark model (RQM) [9] can describe the measurement within uncertainties. The model calculations of Catania [10] and quark (re-)combination mechanism (QCM) [11] both including the coalescence process, also can describe the measurement. In general, PYTHIA8 implemented colour reconnection beyond the leading-colour approximation (CR-BLC) [12] better describes the $\Lambda_{\mathrm{c}}^{+} / \mathrm{D}^{0}$ ratio and $\Sigma_{\mathrm{c}}^{0,++} / \mathrm{D}^{0}$ ratio, which do not contain the strange quarks.

The $\Xi_{\mathrm{c}}^{0} / \mathrm{D}^{0}$ and $\Xi_{\mathrm{c}}^{+} / \mathrm{D}^{0}$ ratio are shown in the left panel of the Fig.2. Most of the model calculations significantly underestimate the $\Xi_{\mathrm{c}} / \mathrm{D}^{0}$ ratio. However, the Catania model describes better the ratios in the measured $p_{\mathrm{T}}$ interval. It means that both fragmentation and coalescence processes are important in pp collisions for the hadronisation process. The $\operatorname{BR}\left(\Omega_{\mathrm{c}}^{0} \rightarrow \Omega^{-} \pi^{+}\right) \times$ $\sigma\left(\Omega_{\mathrm{c}}^{0}\right) / \sigma\left(D^{0}\right)$ ratio is shown in the right panel of the Fig.2. The branching ratio of $\Omega_{\mathrm{c}}^{0} \rightarrow \Omega^{-} \pi^{+}$ is not measured yet, the theoretical calculation of $\operatorname{BR}\left(\Omega_{\mathrm{c}}^{0} \rightarrow \Omega^{-} \pi^{+}\right)$[15] is used to scale the model predictions. Most of the models underestimate the measurements. The Catania model 

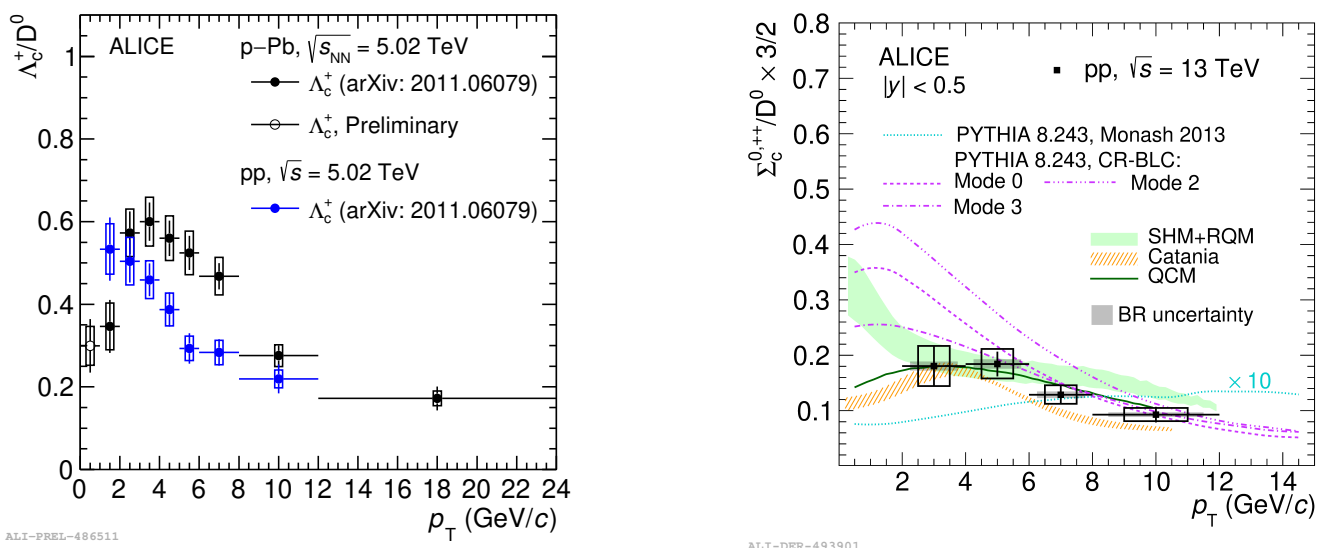

Figure 1: Left panel: $\Lambda_{\mathrm{c}}^{+} / D^{0}$ as a function of $p_{\mathrm{T}}$ in pp and $\mathrm{p}-\mathrm{Pb}$ collisions at $\sqrt{s}=5.02 \mathrm{TeV}$ [13]. Right panel: $\Sigma_{\mathrm{c}}^{0,++} / \mathrm{D}^{0}$ ratio as a function of $p_{\mathrm{T}}$ at $\sqrt{s}=13 \mathrm{TeV}$ [14]. The measurements are compared with model calculations.

is the calculation that gets closer to the measurements of the $\Xi_{\mathrm{c}}^{0} / \mathrm{D}^{0}$ and $\Xi_{\mathrm{c}}^{+} / \mathrm{D}^{0}$ ratio and the $\operatorname{BR}\left(\Omega_{\mathrm{c}}^{0} \rightarrow \Omega^{-} \pi^{+}\right) \times \sigma\left(\Omega_{\mathrm{c}}^{0}\right) / \sigma\left(D^{0}\right)$ ratio.
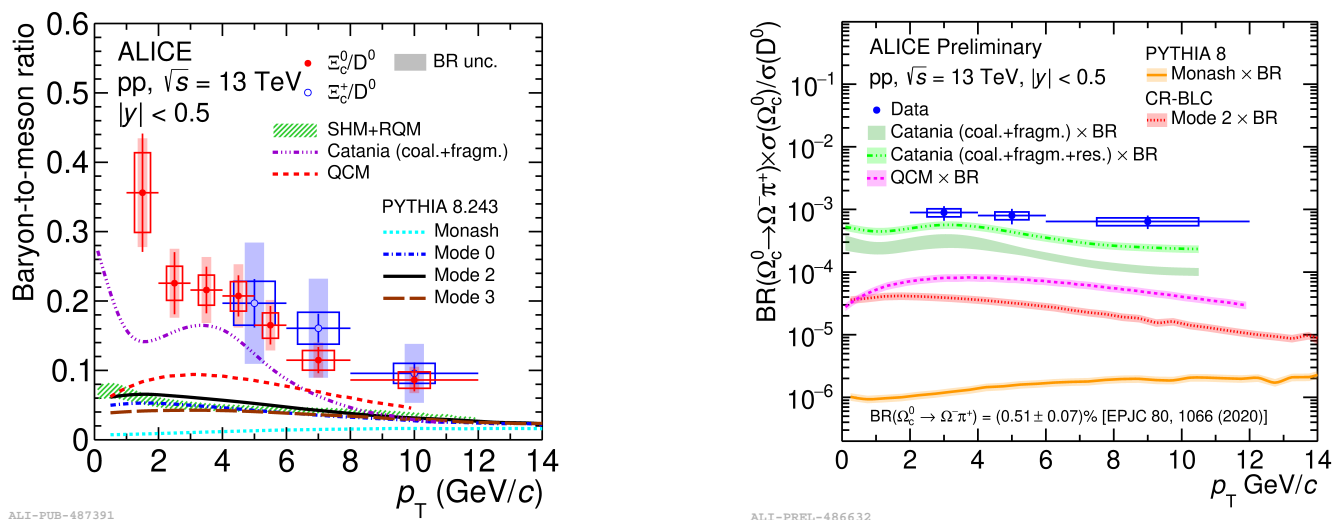

Figure 2: Left panel: $\Xi_{\mathrm{c}}^{0} / \mathrm{D}^{0}$ and $\Xi_{\mathrm{c}}^{+} / \mathrm{D}^{0}$ ratio as a function of $p_{\mathrm{T}}$ at $\sqrt{s}=13 \mathrm{TeV}$ [7]. Right panel: $\operatorname{BR}\left(\Omega_{\mathrm{c}}^{0} \rightarrow \Omega^{-} \pi^{+}\right) \times \Omega_{\mathrm{c}}^{0} / \mathrm{D}^{0}$ ratio as a function of $p_{\mathrm{T}}$ at $\sqrt{s}=13 \mathrm{TeV}$.

\subsection{Charm fragmentation fractions in pp collisions at $\sqrt{s}=5.02 \mathrm{TeV}$}

The charm fragmentation fractions are measured in pp collisions at $\sqrt{s}=5.02 \mathrm{TeV}$. The fragmentation fraction for the $\Xi_{\mathrm{c}}^{0}$ baryon is measured for the first time. The contribution of $\Xi_{\mathrm{c}}^{+}$is considered by doubling the $\Xi_{\mathrm{c}}^{0}$ yield since they are isospin partners. The $\Omega_{\mathrm{c}}^{0}$ is not measured at this energy, so the contribution is included in the systematic uncertainty. The charm fragmentation fractions measured in pp collisions are compared with $\mathrm{e}^{+} \mathrm{e}^{-}$and ep measurements in the left panel of Fig.3. The charm fragmentation fractions measured in pp collisions at the LHC are different from the ones measured in $\mathrm{e}^{+} \mathrm{e}^{-}$and ep collisions, showing that the universality of parton-to-hadron fragmentation is not valid. 
The charm production cross sections at midrapidity per unit of rapidity are shown in the right panel of Fig.3. The charm cross section per unit of rapidity in pp collisions at $\sqrt{s}=5.02 \mathrm{TeV}$ is measured at the LHC for the first time, resulting in $\mathrm{d} \sigma^{\mathrm{c} \overline{\mathrm{c}}} /\left.\mathrm{dy}\right|_{|\mathrm{y}|<0.5} ^{\mathrm{pp}, 5.02 \mathrm{TeV}}=1165 \pm 44(\mathrm{stat})_{-101}^{+134}(\mathrm{syst}) \mu \mathrm{b}$. According to the new measured charm fragmentation fractions, the charm cross section measurements in pp collisions at $\sqrt{s}=2.76 \mathrm{TeV}$ [16] and $7 \mathrm{TeV}$ [17] are updated and are about $40 \%$ higher than the previously published results. The measurements with new charm fragmentation fractions lie at the upper edge of the pQCD calculations $[18,19]$.
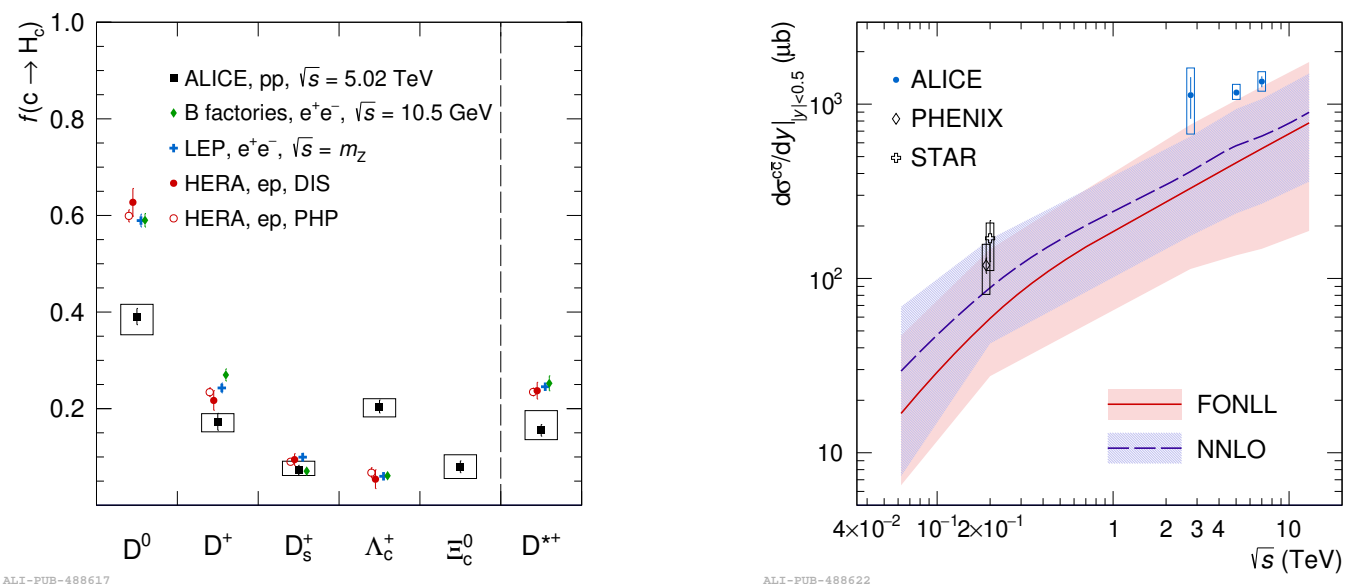

Figure 3: Left panel: Charm-quark fragmentation fractions into charm hadrons in pp collisions at $\sqrt{s}=$ $5.02 \mathrm{TeV}$ compared to the measurements in $\mathrm{e}^{+} \mathrm{e}^{-}$and ep collisions [20]. Right panel: Charm production cross section at midrapidity as function of the collision energy [20].

\section{Summary}

ALICE has measured all single-charm hadron ground states in pp collisions at $\sqrt{s}=5.02 \mathrm{TeV}$ and $\sqrt{s}=13 \mathrm{TeV}$. Furthermore, ALICE measured $\Lambda_{\mathrm{c}}^{+}$in $\mathrm{p}-\mathrm{Pb}$ collisions at $\sqrt{s}=5.02 \mathrm{TeV}$ down to $p_{\mathrm{T}}=0$. Large enhancement of all charm baryons is measured in pp collisions with respect to $\mathrm{e}^{+} \mathrm{e}^{-}$ collisions. The charm fragmentation fractions in pp collisions at $\sqrt{s}=5.02 \mathrm{TeV}$ are measured for the first time, showing showing that charm fragmentation is not universal across collision systems.

\section{References}

[1] ALICE collaboration, $\Lambda_{\mathrm{c}}^{+}$production in pp collisions at $\sqrt{s}=7 \mathrm{TeV}$ and in $\mathrm{p}-\mathrm{Pb}$ collisions at $\sqrt{s_{\mathrm{NN}}}=5.02 \mathrm{TeV}$, JHEP 04 (2018) 108 [1712.09581].

[2] ALICE collaboration, $\Lambda_{\mathrm{c}}^{+}$production and baryon-to-meson ratios in pp and $p$-Pb collisions at $\sqrt{s_{\mathrm{NN}}}=5.02 \mathrm{TeV}$ at the LHC, [2011.06078].

[3] ARGUS collaboration, Observation of the Charmed Baryon $\Lambda_{c}$ in $e^{+} e^{-}$Annihilation at 10-GeV, Phys. Lett. B 207 (1988) 109.

[4] CLEO collaboration, Inclusive production of the charmed baryon $\Lambda_{\mathrm{c}}$ from $\mathrm{e}^{+} \mathrm{e}^{-}$annihilations at $\sqrt{s}=10.55 \mathrm{GeV}$, Phys. Rev. D 43 (1991) 3599. 
[5] M. Arneodo, Nuclear effects in structure functions, Physics Reports 240 (1994) 301.

[6] S. Acharya, D. Adamová, A. Adler, J. Adolfsson, G. Aglieri Rinella, M. Agnello et al., Measurement of beauty and charm production in pp collisions at $\sqrt{s}=5.02 \mathrm{TeV}$ via non-prompt and prompt D mesons, JHEP 2021 (2021) .

[7] ALICE collaboration, Measurement of the cross sections of $\Xi_{\mathrm{c}}^{0}$ and $\Xi_{\mathrm{c}}^{+}$baryons and branching-fraction ratio $B R\left(\Xi_{\mathrm{c}}^{0} \rightarrow \Xi^{-} \mathrm{e}^{+} v_{\mathrm{e}}\right) / B R\left(\Xi_{\mathrm{c}}^{0} \rightarrow \Xi^{-} \pi^{+}\right)$in pp collisions at $\sqrt{s}=13 \mathrm{TeV}$, [2105.05187].

[8] P. Skands, S. Carrazza and J. Rojo, Tuning PYTHIA 8.1: the Monash 2013 Tune, Eur. Phys. J. C 74 (2014) 3024 [1404 . 5630].

[9] A. Andronic, F. Beutler, P. Braun-Munzinger, K. Redlich and J. Stachel, Statistical hadronization of heavy flavor quarks in elementary collisions: Successes and failures, Phys. Lett. B 678 (2009) 350 [0904 . 1368].

[10] V. Minissale, S. Plumari and V. Greco, Charm hadrons in pp collisions at lhc energy within a coalescence plus fragmentation approach, Phys. Lett. B 821 (2021) 136622.

[11] J. Song, H.-h. Li and F.-1. Shao, New feature of low $p_{\mathrm{T}}$ charm quark hadronization in pp collisions at $\sqrt{s}=7$ TeV, Eur. Phys. J. C 78 (2018) 344 [1801.09402].

[12] J.R. Christiansen and P.Z. Skands, String Formation Beyond Leading Colour, JHEP 08 (2015) 003 [1505.01681].

[13] ALICE collaboration, $\Lambda_{\mathrm{c}}^{+}$production in pp and in $p$ - $P b$ collisions at $\sqrt{s_{\mathrm{NN}}}=5.02 \mathrm{TeV}$, [2011.06079].

[14] ALICE collaboration, Measurement of prompt $D^{0}, \Lambda_{c}^{+}$, and $\Sigma_{c}^{0,++}(2455)$ production in $p p$ collisions at $\sqrt{s}=13 \mathrm{TeV},[2106.08278]$.

[15] Y.-K. Hsiao, L. Yang, C.-C. Lih and S.-Y. Tsai, Charmed $\Omega_{c}$ weak decays into $\Omega$ in the light-front quark model, Eur. Phys. J. C 80 (2020) .

[16] B. Abelev, J. Adam, D. Adamová, A.M. Adare, M.M. Aggarwal, G. Aglieri Rinella et al., Measurement of charm production at central rapidity in proton-proton collisions at $\sqrt{s}=2.76 \mathrm{TeV}, \mathrm{JHEP} 2012$ (2012).

[17] ALICE collaboration, Measurement of D-meson production at mid-rapidity in pp collisions at $\sqrt{s}=7$ TeV, Eur. Phys. J. C 77 (2017).

[18] M. Cacciari, M. Greco and P. Nason, The $p_{\mathrm{T}}$ spectrum in heavy flavor hadroproduction, JHEP 05 (1998) 007 [hep-ph/9803400].

[19] D. d'Enterria and A.M. Snigirev, Triple parton scatterings in high-energy proton-proton collisions, Phys. Rev. Lett. 118 (2017) 122001.

[20] ALICE collaboration, Charm-quark fragmentation fractions and production cross section at midrapidity in pp collisions at the LHC, [2105.06335]. 